\title{
Archivos coloniales y el ARTe de gobernar
}

\author{
AnN LAURA STOLER \\ PROFESORA UNIVERSITARIA DISTINGUIDA DE ANTROPOLOGÍA Y ESTUDIOS HISTÓRICOS, \\ Nueva Escuela de Investigación Social, Nueva YorK \\ StolerA@newschool.edu
}

\begin{abstract}
Resumen
os antropólogos dedicados a los estudios poscoloniales adoptan cada vez más una Lperspectiva histórica y recurren a los archivos, aunque esta actividad tiende a ser más extractiva que etnográfica. Los documentos son todavía fragmentos que se examinan en silencio para confirmar la invención de ciertas prácticas coloniales o recalcar reivindicaciones culturales. Sin embargo, la extracción del contenido de comisiones, informes y otras fuentes rara vez presta atención a su disposición y forma particular. Es necesario cambiar el enfoque del archivo-como-fuente al archivo-como-objeto. Este artículo, sostiene que los archivos se deben considerar no como lugares de recuperación del conocimiento, sino de producción de conocimiento, como documentos legales y sitios de la etnografía del Estado. Este enfoque exige un compromiso continuo con los archivos como agentes culturales de producción de "hechos", de taxonomías en desarrollo y de la autoridad estatal. Aquello que constituye un archivo, qué forma adopta y qué sistemas epistemológicos y de clasificación señalan el camino en momentos específicos que conforman (y reflejan) las características esenciales de la política y el poder estatal colonial.
\end{abstract}

PALABRAS Clave: archivos, archivística, burocracia, archivos coloniales, etnografía, conocimiento.

\section{Archives and the Arts of Governance}

\begin{abstract}
Anthropologists engaged in postcolonial studies are increasingly adopting an historiAcal perspective and using archives. Yet their archival activity tends to remain more extractive than ethnographic. Documents are thus still invoked piecemeal to confirm the colonial invention of certain practices or to underscore cultural claims, silent. Yet such mining of the content of government commissions, reports, and other archival sources rarely pays attention to their peculiar placement and form. Scholars need to move from archive-as-source to archive-as-subject. This article, argues that scholars should view archives not as sites of knowledge retrieval, but of knowledge production, as monuments of states as well as sites of state ethnography. This requires a sustained engagement with archives as cultural agents of "fact" production, of taxonomies in the making, and of state authority. What constitutes the archive, what form it takes, and what systems of classification and epistemology signal at specific times are (and reflect) critical features of colonial politics and state power.

KEYWORDS: archives, archiving, bureaucracy, colonial archives, ethnography, knowledge
\end{abstract}

\section{Revista Colombiana de Antropología}

Volumen 46 (2), julio-diciembre 20I0, pp. 465-496 
La genealogía es gris, meticulosa y pacientemente documentalista. Trabaja sobre áreas de pergaminos embrollados y confusos; sobre documentos garabateados y muchas veces reescritos.

(Foucault, M., 1977, p. 139)

s ste ensayo ${ }^{1}$ trata sobre el orden colonial de las cosas, tal y como se aprecia a través la producción de archivos. En él se investiga qué se podría conocer en profundidad sobre lo colonial considerando no solo el contenido de los archivos coloniales, sino

I. Artículo publicado originalmente con el título Colonial Archives and the Arts of Governance en la revista Archival Science 2, 8I-IO9, 2002. Licencia de traducción de Springer para el Instituto Colombiano de Antropología e Historia No. 2373011050167.

2. Sobre el "giro historico", véase la introducción a T. J. McDonald (I966). Este ensayo representa una versión sucinta del capítulo 1 del libro Along the Archival Grain: Epistemic Anxieties and Colonial Common Sense. Princeton: Princeton University Press, 2008. Algunos de sus fragmentos se basan en las lecturas L. H. Morgan (I996). Existe una versión diferente de este documento en el texto editado por C. Hamilton, Harris, V., Taylor, J., Pickoever, M., Reid, G. y Saleh (eds.) (2002). Refiguring the Archive. (pp. 83-IO2). Dordrecht, Boston, London. Kluwer Academic Publishers.

su forma particular y a veces peculiar. Este documento se centra en el archivo como proceso, más que en los archivos como objetos. Se consideran los archivos como experimentos epistemológicos y no como meras fuentes y los archivos coloniales como muestras representativas del conocimiento puesto a prueba. Más importante aún, este ensayo considera los archivos coloniales como transparencias en las que se inscriben relaciones de poder y como tecnologías de gobierno que son intrincadas en sí mismas. En este escrito se contemplan dos problemas: situar los nuevos enfoques sobre los archivos coloniales dentro del "giro histórico" de las últimas dos décadas, $y$, por otra parte, proponer historias coloniales importantes que se deben desarrollar al prestar mayor atención a una política del conocimiento que reconozca la importancia de los géneros archivísticos, las culturas de documentación, las ficciones de acceso y las convenciones archivísticas. ${ }^{2}$

\section{Escepticismo EPISTEMOLÓGICO, ARCHIVOS Y EL “GIRO HISTÓRICO”}

uatro décadas después de la desatendida advertencia del
antropólogo social británico, E.E. Evans-Pritchard, de que
la antropología tendría que escoger entre ser histórica o no 
ser nada, y de que C. Lévi-Strauss objetara que a la historia no se le ha conferido ningún "valor especial" ni un espacio analítico privilegiado, los estudiosos de la cultura han aceptado un desafío transformador al celebrar con un gusto sin precedentes lo que se ha denominado "el giro histórico" (Evans-Pritchard, E.E., I95I, p. I52; Lévi-Strauss, C., I966, p. 256). Algunos podrán sostener que la relación de la antropología con la historia de las últimas dos décadas, a diferencia del reciente "giro" de otras disciplinas, no se trata del todo de un giro, sino de un regreso a sus principios elementales, esto es, una investigación dentro de procesos acumulativos de producción cultural, pero sin las aspiraciones tipológicas y las presunciones evolutivas que una vez se aceptaron. Otros podrán contestar que el giro febril hacia la historia representa un punto de partida importante de una empresa temprana, una ruptura más explícita con la complicidad de larga data de la antropología en la política colonial. ${ }^{3}$ De esta forma, se podría argumentar que el giro histórico no señala un giro hacia la historia en sí, sino una reflexión diferente sobre la política del conocimiento: un rechazo a las categorías y distinciones culturales que una vez invistieron las normas imperiales y en las que se basan aún las prácticas estatales poscoloniales.

El compromiso con los usos y abusos del pasado permea muchas disciplinas académicas, pero ninguna tanto como esta área floreciente de la etnografía colonial. En la última década, los estudiosos de lo colonial se han enfrentado a las categorías, los marcos conceptuales y las prác-

3. Para encontrar alguna explicación sobre el rango de las diferentes agendas del "giro histórico" actual, véase N. B. Dirks, G. Eley, y S. B. Ortner, (I994) y T. J. McDonald (I996); específicamente sobre la historia en la imaginación antropológica, véase G. Sider y G. Smith (I997). Véase también R. Fox (I99I) y J. Faubion (I993).

4. Véase, por ejemplo, las introducciones y los ensayos en N. Dirks (I992) y F. Cooper y A. L. Stoler (1997). ticas de las autoridades coloniales y sus estados taxonómicos. ${ }^{4}$ El cuestionamiento de la formación del conocimiento colonial y de las categorías sociales privilegiadas que genera ha reformado aquello que los estudiosos de lo colonial consideran fuentes de conocimiento y lo que esperan de ellas. La atención prestada a los dominios íntimos en que intervinieron los Estados coloniales ha llevado a reconsiderar lo que consideramos como las bases de la autoridad europea y sus tecnologías fundamentales (Stoler, A., 2002). Al abordar el colonialismo como una historia viva que informa y moldea el presente y no como un pasado acabado, una nueva generación de académicos ha aceptado la invitación de 
M. de Certeau de "sondear" los nuevos terrenos a medida que reimaginan qué clases de conocimiento situado han producido tanto las fuentes coloniales como sus lugares respectivos en la "operación historiográfica” (de Certau, M., I988). Algunos estudiosos del colonialismo releen tales archivos y elaboran historias orales con las personas que vivieron los eventos plasmados en ellos para comentar sobre sus narrativas coloniales (Price, R., I998; Stoler, A.L. y Strassler, K., 2000,

5. Sobre el poder de las imágenes en la creación de la normas coloniales, véase E. Edwards, I997.
White, L., 2000). Otros hacen lo mismo con fotografías, grabados y arte documental. ${ }^{5}$ Algunos se ocupan de la manera como se han solicitado y reciclado los documentos coloniales para ratificar antiguos derechos o para presentar nuevas demandas políticas. Como parte de un movimiento más amplio, ya no estudiamos cosas, sino su elaboración. Los analistas del colonialismo dentro y fuera de la antropología destinan hoy parte de su tiempo en repensar lo que constituye el archivo colonial, así como en reconsiderar de qué manera se enfrentan los documentos escritos y convergen con las memorias coloniales en el campo poscolonial.

Si hace 30 años la advertencia de E. E. Evans-Pritchard de que "los antropólogos se han inclinado por la poca rigurosidad en el manejo de las fuentes documentales" tuvo poca resonancia, ahora la tiene más. Porque por más profundo y completo que haya sido el giro archivístico en los estudios poscoloniales de los años 9o, lo más sorprendente es lo sutil y tentativo que puede permanecer (Evans-Pritchard, E.E., I96I, p. 5). Los antropólogos pueden dejar de ver los archivos como una cuestión de otras disciplinas y puede que estos archivos dejen de ser tratados como sitios inertes de almacenamiento y conservación (Ginzburg, C., I989b). Sin embargo, el trabajo en archivo tiende a ser aún una empresa más extractiva que etnográfica. Los documentos se siguen citando en fragmentos y seleccionando para confirmar la invención colonial de prácticas tradicionales o para recalcar reivindicaciones culturales.

La antropología nunca se ha comprometido con "agotar" las fuentes, como B. Cohn una vez criticó a los historiadores por hacerlo con tal fervor moral. Sin embargo, la metáfora extractiva sigue siendo pertinente en ambos casos (Cohn, B., 1980). Los estudiosos de las experiencias coloniales "extraen" el contenido de las comisiones e informes gubernamentales, pero rara vez 
prestan atención a su forma o contexto peculiar. Nosotros nos centramos en documentos ejemplares en vez de hacerlo en la sociología de las copias o de las reivindicaciones de verdad que se alojan en la memorización y la repetición. Con cautela citamos ejemplos de los excesos coloniales, si no con malestar por el patetismo y voyerismo que tales citaciones entrañan. Podemos burlarnos con facilidad del fetichismo de la labor de los historiadores, pero subsiste la convicción compartida de que el acceso a lo "clasificado" y "confidencial" son los hallazgos que ambicionan los trabajos intelectuales convincentes e ingeniosos. ${ }^{6} \mathrm{La}$ capacidad de lograrlos mide el mérito académico. Y no es menos la convicción compartida de que
6. Sobre los viajes hacia los archivos como "hazañas de la destreza [del hombre]" en la cultura de la clase media del siglo XIX, véase B. G. Smith, 1995 . tales tesoros guardados son los lugares donde en realidad se almacenan los secretos del Estado colonial.

Hay varias maneras de contextualizar la clase de desafíos que tengo en mente, pero al menos una parece evidente: inmersos como los estudiosos de la cultura lo han estado al abordar las etnografías como textos, ahora reflexionamos de manera crítica sobre la elaboración de documentos y sobre cómo decidimos utilizarlos, sobre los archivos no como lugares de recuperación del conocimiento, sino de producción del mismo, como monumentos estatales y sitios para la etnografía del Estado. Esto no constituye un rechazo a los archivos coloniales como fuentes del pasado. Más bien, apunta hacia un compromiso constante con tales archivos como artefactos culturales de producción de hechos, de taxonomías en el hacer y de diversas nociones sobre lo que ha configurado la autoridad colonial.

Así como R. Guha y G. Dening han aseverado por mucho tiempo, las "fuentes" no son "manantiales de significado real" ni "pilas" de verdades coloniales en sí mismas (Guha, R., I994, pp. 336-37I; Dening, G., 1995, p. 54). Aunque los documentos sean fidedignos, auténticos y confiables todavía despiertan preguntas. Sin embargo, prestar atención a las condiciones sociales y políticas que produjeron estos documentos, aquello que C. Ginzburg ha denominado sus "paradigmas probatorios", ha alterado el sentido de lo que la confianza y la veracidad podrían indicar e implicar en lo político. La tarea consiste menos en distinguir la ficción de los hechos, que en rastrear la realización y consumo de estos "hechos" en sí. Con este movimiento, los estudios coloniales 
toman un nuevo rumbo hacia la exploración de las coordenadas de inteligibilidad que produjeron tales "paradigmas probatorios" en un momento particular, para un contingente social particular y de una forma particular (Ginzburg, C., I989a, pp. 96-I25).

Los estudiosos de lo colonial han llegado a ver que las apropiaciones de la historia colonial se infunden en las agendas políticas, de tal manera que unas historias se pueden elegir para la práctica histórica y otras no (Cohen, D. W., I992). Preguntas problemáticas sobre la manera como los recuerdos personales toman forma y se borran gracias a los Estados han enfatizado en el análisis de cómo las prácticas del pasado se depuran para usos y proyectos futuros (Nuttall, S. y Coetzee, C.I998; Rappaport, J., 1994). Tales investigaciones invitan a retornar a la documentación en sí, a la labor "pedagógica" que la raíz latina docere entraña, a qué y quién se educa dentro de la reorganización burocrática de fórmulas repetitivas, maquinaciones genéricas y digresiones prescriptivas que conforman la mayor parte del archivo colonial. La cuestión de la "parcialidad" oficial da lugar a un desafío distinto: identificar las condiciones de posibilidad que dieron forma a aquello que se podía escribir, a las repeticiones que se autorizaban, a la definición de las competencias consideradas meritorias en la escritura de archivos, historias que no se podían contar y qué se podía decir. A. Ashforth pudo exagerar esto en su estudio sobre la Comisión de Asuntos Nativos de Sudáfrica, cuando dijo que "el sello real del poder" en los Estados modernos es "el escritorio, el lugar de la escritura", pero puede que no haya estado lejos de la realidad (Ashforth, A., I990, p. 5). El hecho de que cada documento venga en capas y dé cuenta de los acontecimientos anteriores y de la semántica cultural de un momento político esclarece el punto. Aquello que constituye un archivo, la forma que adopta y los sistemas de clasificación utilizados en momentos específicos constituyen la esencia verdadera de la política colonial. 
Volumen 45 (2), julio-diciembre 2010

\section{DE LA EXTRACCIÓN A LA ETNOGRAFÍA EN LOS ARCHIVOS COLONIALES}

La transformación de la actividad archivística es el punto de partida y la condición de la nueva historia

(De Certeau, M., I988, p. 75).

Ci alguien pudiese decir que los archivos fueron tratados por los historiadores alguna vez como el medio para llegar a un fin, esto ya no corresponde a la realidad actual. El placer de "un cuarto con un buen acervo de manuscritos, facilidad de acceso y un aura de aislamiento silencioso" es cosa del pasado. ${ }^{7}$ Durante la última década, el escepticismo epistemológico ha tomado por asalto a los estudios culturales e históricos. El énfasis en la historia como narrativa y en la escritura histórica como un acto políticamente cargado ha hecho que pensar en los archivos ya no sea la preocupación ordinaria de historiadores que solo realizan "trabajos preliminares" o de archivistas sin preparación, tampoco el requisito de entrada de neófitos obligados a demostrar que dominan las herramientas del oficio. El "archivo" ha sido elevado a un nuevo estatus teórico, con suficiente prestigio para garantizar buenos réditos y merecedor de un examen cuidadoso en sí mismo. En Archive Fever, J. Derrida captura de manera convincente tal impulso dándole nombre y proporcionando un léxico explí-

7. Una frase que utiliza J. Sherron De Hart para recalcar las "problemáticas de evidencia" en la reconstrucción histórica contemporánea (Sherron de Hart, J., 1993, p. 582).

8. Véase también D. LaCapra (1995, p. 807) donde advierte que el "problema de la lectura de los archivos es cada vez más un asunto de aquellos que realizan investigación en archivos". cito y evocativo para su legitimación en la teoría crítica (Derrida, J., 1995). Sin embargo, en Fiction in the Archives, de N. Zemon Davis; Myth and Archive, de R. González Echevarría; Imperial Archive, de T. Richards; y Archives Interdites, de S. Coombe, para nombrar unos cuantos, se sugiere que el destello de J. Derrida vino después de ocurrido el giro archivístico. ${ }^{8}$

Este movimiento de los archivos-como-fuente a los archivoscomo-objeto gana su aceptación en el mundo contemporáneo a partir de una serie de cambios analíticos, problemas prácticos y proyectos políticos. Para algunos, como en las matizadas incursiones archivísticas de G. Dening, representa un retorno a la meticulosa “poética del detalle” (Dening, G., I995). Para otros, como M. R. 
Trouillot en el tratamiento que da a los silencios de los archivos de la Revolución Haitiana, y D. W. Cohen en sus "exploraciones de la historia”, el archivo-como-objeto señala un nuevo enfrentamiento con la producción histórica, esto es, qué narraciones se autorizan, qué procedimientos se necesitaron y qué es posible conocer acerca del pasado (Trouillot, M. R., I994 y I995). Para B. Smith, la investigación en archivos, como el seminario universitario, eran los lugares del siglo XIX donde la ciencia histórica era marcada con credenciales del género (Smith, B.G., 1995). Desde luego que los archivistas también han pensado acerca de la naturaleza e historia de los archivos por algún tiempo (Cook, T., 1997; Duchein, M., I992; Posner, E., I984). Aquello que marca este momento es la profusión de foros en los que historiadores se unen a archivistas en nuevas conversaciones acerca de la evidencia documental, la conservación de registros y la teoría archivística (Berner, R., I983; Cook T., I992; Foote K.E., I990; O’Toole, J. M., I994). ${ }^{9}$ Ambos se preocupan por las políticas de almacenamiento, la definición de cuál información es relevante y qué se debe mantener en los archivos, a medida que las compilaciones en papel dan lugar a las formas digitales (Cook, T., I994).

En la teoría cultural, "el archivo" tiene una "A" mayúscula, es figurativo y lleva a cualquier otra parte. Puede que no represente un lugar material ni un conjunto de documentos, sino que sirva como metáfora contundente de cualquier corpus de olvidos y colecciones seleccionados y, con igual importancia, de las seducciones y anhelos que entrañan tales búsquedas y acumulaciones de lo primario, lo originario y lo inexplorado. ${ }^{10}$ Para aquellos que se han inspirado de manera más directa en la Arqueología del

9. En cuanto al significado de los cambios en cómo los archivistas han enmarcado su trabajo en los últimos 15 años, véanse muchos de los artículos en The American Archivist y Archivaria.

IO. Esta dirección metafórica es más evidente en los aportes de las dos ediciones especiales de History of the Human Sciences dedicadas a "The Archive", II(4) (noviembre de I998) and I2(2) (mayo de 1999). La valorización de J. Derrida del "archivo" como imaginario y metáfora predomina en ambos. Sobre el archivo como metáfora, véase también A. Sekula (I986). saber, de M. Foucault, el archivo no es una institución, sino "la ley de lo que puede ser dicho". No es una biblioteca de eventos, sino "el sistema que rige la aparición de los enunciados como eventos y cosas singulares", el "sistema de lo que puede ser enunciado" en (Foucault, M., I972, parte III, pp. 79-I34).

Desde cualquier punto de vista (y hay más que los mencionados) el "giro archivístico" registra un replanteamiento de la materialidad y el imaginario de las colecciones y señala qué 
reivindicaciones de verdad yacen en la documentación (Geary, P., I994, pp. 8I-II4). Tal "giro archivístico" converge con una profusión de nuevos trabajos en la historia de la ciencia, que no tratan sobre los archivos ni en sentido figurado ni literal. Pienso en este punto en los estudios de I. Hacking sobre la historia política de la teoría de las probabilidades y las inversiones estatales en "el dominio de las probabilidades"; en el análisis que hace S. Shapin de la historia social de las verdades científicas, en el que hace seguimiento al poder de predecir y lo encuentra como aquel que disfrutan hombres cultos y confiables y que está reservado para ellos; en la obra de M. Poovey sobre cómo la noción del "hecho moderno" era producida históricamente; en el estudio de A. Desrosieres (entre muchos otros) sobre la estadística como ciencia del Estado y el de S. Patriarca sobre la estadística como un modo de representación moderno; en el análisis de L. Daston del desa-

II. Sobre el poder de las "expresiones persuasivas" en la creación de dogmas científicos, véase C. Norris, (1995) y B. Anderson, (1991). rrollo de la teoría clásica de la probabilidades como medio para medir las incertidumbres de un mundo en proceso de modernización (Desrosieres, A., I998; Hacking, I. , I990; Patriarca, S., I998; Poovey, M., I998;Shapin, S., I994). ${ }^{11}$ También se podrían sumar los ensayos de A. Grafton sobre los pies de página como líneas que llevan a comunidades morales y sus principios de autoridad y verdad (Grafton, A., 1997).

¿Qué tienen en común estos estudios? Todos están relacionados con las coordenadas sociales que legitiman las epistemologías: cómo imaginan las personas que saben lo que saben, qué instituciones validan ese conocimiento y cómo lo hacen. Ninguno aborda las convenciones y categorías de análisis (estadísticas, hechos, verdades, probabilidades, notas al pie, etc.) como inocuas o propicias. Estos estudios coinciden en las preguntas acerca de las normas de fiabilidad y veracidad, en los criterios de credibilidad y en qué proyectos morales y previsibilidades políticas atienden estas convenciones y categorías. Todos formulan un conjunto similar de preguntas históricas acerca del conocimiento aprobado y el poder: qué fuerzas políticas, indicios sociales y virtudes morales producen conocimientos adecuados que, a su vez, descalifican otras formas de conocimiento y otros conocimientos. Desde mi perspectiva, no hay otro conjunto de problemas que revista mayor importancia para la política colonial de los archivos y sus Estados (padres) archivistas. 
El giro archivístico también se puede rastrear en otros dominios territoriales, lo que indica que algo similar a la etnografía, a manera de archivo, ha rondado por algún tiempo. El análisis microhistórico de C. Ginzburg sobre un molinero del siglo XVI, así como el manejo que N. Davis hace de las narraciones del perdón en Fiction in the Archives, se basa en documentos "hostiles" de las élites para develar "la diferencia que existe entre la imagen que subyace bajo los interrogatorios de jueces y el testimonio real de los acusados" (Ginzburg, C., I982, pp. xvii, xviii). Ninguno de estos trabajos fue concebido como etnografías del archivo, pero ambos apuntan en tal dirección. La atención que N. Davis presta a "la forma como las personas relatan las historias, lo que consideran una buena historia, cómo dan cuenta de las intenciones" muestra que las cartas de indulgencia del siglo XVI dicen más que los simples hechos relatados por los campesinos (Davis, N., I987, p. 4). Las narraciones del perdón también registran las "limitaciones de la ley", el monopolio del poder real en la justicia pública y la indulgencia que la monarquía profesaba cada vez más (Davis, $\mathrm{N}$. Z., I987, p. 4). Fiction in the Archives ofrece relatos amoldados que dan señales de verdades morales basadas en metáforas compartidas y una elevada cultura literaria que dependen del poder estatal y de las inscripciones archivadas de su autoridad.

Si bien los participantes recientes en el giro archivístico se han sentido atraídos por el argumento de J. Derrida de que "no existe poder político sin el control de los archivos", la insistencia en la relación entre lo que cuenta como conocimiento y quien tiene el poder ha sido por largo tiempo un principio fundamental de la etnografía colonial (Derrida, J., I995, p. 4). La insistencia de M. R. Trouillot en su estudio sobre la Revolución Haitiana de que "las narrativas históricas tienen como premisa acuerdos anteriores, que se asientan a su vez en la distribución del poder archivístico", le permite seguir las huellas desvanecidas y los silencios impuestos a los cuales las personas se han desplazado y así ir más allá (Trouillot, M. R., I995, p. 55). La observación de N. Dirk de que las primeras historiografías coloniales de la India Británica —que más adelante se expresaron en papel— dependían de los informantes nativos, llama nuestra atención sobre la relación entre la archivística, los expertos y la producción de conocimiento (Dirks, N.B., 1993). El énfasis más reciente de C. Bayly en las formas en que el servicio de inteligencia británico en la India colonial trabajó por medio de canales de información 
nativos sitúa el acceso estatal a la "información" como un nodo en el arte del gobierno y como terreno muy contestado (Bayly, C., 1996). Mi propio trabajo sobre "las jerarquías de la credibilidad" contenidas en las narrativas coloniales de las Indias Holandesas, ya que estas restringían cuáles eran los relatos plausibles, examina las políticas coloniales a partir de las "famosas" distribuciones de la producción documental del Estado y a través de los rumores (difundidos por la población nativa sitiada) que se tejieron a través de dicha producción (Stoler, A. L., I992b).

Tal y como M. Foucault advirtió en tono provocativo, el archivo no es la suma de todos los textos que preserva una cultura ni las instituciones que permiten preservar tales registros. El archivo es más bien ese "sistema de enunciados", aquellas "normas de la práctica”, que moldean las regularidades específicas de aquello que se puede y no se puede decir (Foucault, M., I972, pp. 79-I34). Los estudiosos de lo colonial han lidiado con esta formulación para capturar aquello que hace de los archivos coloniales documentos excluyentes y monumentos que expresan configuraciones de poder particulares.

Tanto G. González Echevarría como T. Richards siguen a M. Foucault al abordar el archivo imperial como "la representación fantástica de un modelo epistemológico” (Foucault, M., I993, p. II). Para T. Richards, el archivo es material y figurativo, una metáfora de una imaginación imperial británica insatisfecha pero compartida. El archivo imperial era la tecnología estatal suprema de finales del siglo XIX y el prototipo diciente del Estado posmoderno, proclamado sobre la dominación mundial de la información y los circuitos a través de los que circulan los "datos". G. González Echevarría ubica los archivos como reliquia y ruina, un repositorio de creencias codificadas, géneros literarios que testifican, grupos de conexiones entre lo secreto, el poder y la ley (González, G., I99o, p. 30). Para él fueron los discursos legitimadores de los archivos coloniales españoles los que dieron a la novela latinoamericana su contenido específico y forma temática. Tanto para T. Richards como para G. González Echevarría, el archivo es un patrón que decodifica algo más. Ambos nos obligan a pensar de manera diferente las ficciones de los archivos, pero reservan su análisis en detalle para la literatura y no para los archivos coloniales en sí. ${ }^{12}$

I2. Así pues, para T. Richards, Lost Horizon de Hilton y Kim de Kipling son entradas en un archivo victoriano que constituyó "el prototipo del sistema mundial de dominación a través de la circulación, un aparato para controlar los territorios mediante la producción, distribución y consumo de información sobre él". 
La verdadera pregunta no es si el "archivo" debe tratarse como un conjunto de normas discursivas, un proyecto utópico, un depósito de documentos, un corpus de enunciados o todas las anteriores. Los archivos coloniales eran a la vez lugares

I3. Este vínculo entre el poder del Estado y lo que se considera historia fue establecido hace un tiempo por F. Hegel en The Philosophy of History, como H. White señala: "El Estado es el que por vez primera presenta un contenido que no solo es apropiado para la prosa de la historia, sino que la engendra en su propio ser". (White, H., I987 p. I2). del imaginario e instituciones que creaban historias a medida que ocultaban, revelaban y reproducían el poder estatal. ${ }^{13} \mathrm{El}$ poder y el control, como muchos académicos han señalado, son fundamentales para la etimología del término (González Echevarría, G., I990, p. 3I). Del latín archivum, "residencia del magistrado" y del griego arkhe, ordenar o gobernar, los archivos coloniales ordenaban (tanto en el sentido imperativo como en el taxonómico) los criterios de la evidencia, pruebas, testimonios y testigos que servían para elaborar narraciones morales. La "narración de hechos", las historias morales y las múltiples versiones - características que $\mathrm{H}$. White atribuye a lo que cuenta como historia- determinan qué argumentos específicos "funcionaron" también en los archivos coloniales (White, H., I987, pp. 26-57). El Estado colonial utilizó las historias factuales con el fin de afirmar para sí las ficciones contenidas en ellas; con los relatos morales trazó el alcance de sus misiones filantrópicas y los relatos culturales fueron desacreditados o reivindicados en múltiples y controvertidas versiones.

Desde esta perspectiva, es claro que los archivos del siglo XIX y principios del XX durante la administración holandesa de las Indias no fueron leídos de manera nada azarosa. Ciertos asuntos revistieron importancia por la manera como se categorizaban y por el discurso en que se enmarcaban. Los intercambios oficiales entre el gobernador general y sus subordinados, entre el gobernador general y el ministro de las colonias, y entre el ministro y el rey, sirvieron como punto de referencia para el pensamiento administrativo. Organizadas en folios, las portadas presentaban largas listas de dossiers con referencias cruzadas y decisiones sobre genealogías resumidas de lo que se constituía como importante, precedente y como "razones de Estado". Estos folios, que traían evidencias anexas que podían incluir el testimonio de expertos e informes de comisiones, contenían y confirmaban qué se consideraba como prueba y quién copiaba a quién en la cadena de mando. La atención prestada a momentos de des- 
confianza y dispersión, reveses del poder e incumplimientos contractuales fueron durante un tiempo la característica propia de una historia política y social crítica. Lo que ha cambiado es la apreciación de la medida en que las prácticas archivísticas de estos "imperios de papel" indican los cambios en sus tecnologías de gobierno. ${ }^{14}$

Aunque sea evidente que los archivos coloniales son producto de la maquinaria estatal, no lo es tanto que sean, por mérito propio, tecnologías que fortalecen la producción de esos mismos Estados (Stoler, A.L., I997). Los sistemas de registros escritos fueron producto de instituciones, pero los restos de documentos (informes semanales a superiores, resúmenes de informes, recomendaciones basadas en informes) requirieron un sistema complejo de codificación a través del cual se pudieran rastrear. El arte colonial de gobernar se construyó sobre bases de estadísticas y encuestas, pero también fuera del aparato administrativo que producía tal información. Varios circuitos de comunicación (líneas de transporte, servicios de correo y telégrafos) eran financiados por las arcas del Estado y los sistemas tributarios que las mantenían repletas. Las casas editoriales coloniales se aseguraron de que los documentos fueran selectivamente duplicados, diseminados o destruidos. Los edificios de oficinas coloniales se construyeron para asegurar que los documentos serían catalogados y almacenados de forma apropiada. Y de manera similar al régimen racial general en el que los archivos se producían, los jóvenes "mestizos", los “indonesios", que estaban vedados para ascender a otros cargos de servicio civil, fueron los escribas que hicieron que el sistema funcionara. Empleados como escribientes y copistas de la burocracia colonial, se les denominaba comúnmente "máquinas copiadoras", y luego eran desdeñados por su falta de iniciativa, su poco dominio del holandés y su fácil adaptación a tales funciones de imitación y degradación. La atención prestada a este tipo de andamiaje del Estado colonial se traduce en una lectura etnográfica de los archivos muy diferente de lo que eran las historias del régimen colonial varias décadas atrás. 


\section{SIGUIENDO LA CORRIENTE ARCHIVÍSTICA}

Ci se intentara caracterizar aquello que ha inspirado un enfoque crítico hacia los archivos coloniales durante los últimos I5 años, sería un compromiso ante la idea de leer los archivos coloniales "a contracorriente". Los estudiosos de lo colonial, inspirados en la economía política, fueron educados para escribir historias populares "desde abajo", historias de resistencia que podrían situar la agencia humana en pequeños gestos de rechazo y silencio entre los colonizados. ${ }^{15}$ De esta manera, el estudio de los archivos coloniales fue consagrado a la lectura de "fuentes de las clases altas desde abajo", con el fin de revelar el lenguaje normativo y los sesgos inherentes a las percepciones estatistas (Stoler, A.L., 1985).

El proyecto político consistía en escribir las historias que el Estado no cuenta y que podrían demostrar la realidad distorsionada del conocimiento oficial y las consecuencias duraderas de tales distorsiones políticas. De acuerdo con la formulación de R. Guha, los documentos coloniales eran patrañas retóricas manuscritas en las que se suprimieron hechos sobre la subyugación, se reclasificaron los crímenes insignificantes como subversiones

I5. Para una relación más detallada de estos cambios, véase el nuevo prefacio de mi libro Capitalism and Confrontation in Sumatra's Plantation, I870-I979.

I6. Para ver una versión reciente y sofisticada de este proyecto de selección, véase S. Amin (I995). políticas o solo se desaparecía a los colonizados. Los intereses políticos eran puestos en las tácticas analíticas de inversión y recuperación: un esfuerzo por reubicar a aquellas personas objeto de la disciplina colonial como sujetos subalternos y agentes de prácticas, que tomaron decisiones por sí mismos (aunque obligados). En este contexto, los documentos de archivo hacen contrapeso a la etnografía, pero no la reemplazan. ${ }^{16}$

Sin embargo, la autoridad colonial y las prácticas que la sostenían penetraron en lugares más diversos que aquellos que iban tras este "romance de resistencia" una vez imaginado. Si en estas primeras iniciativas de elaboración de historias de acción popular se hubiera conocido la aseveración de K. Marx de que "las personas construyen su propia historia, pero no exactamente como la desearían”, en ellas también se enfatizaría que el 
gobierno colonial reposa en más que las inequidades calculadas de relaciones específicas de producción e intercambio. Al mirar con mayor detenimiento las representaciones culturales de poder cuidadosamente establecidas, los estudiosos del mundo colonial han puesto su atención en las prácticas que privilegiaron ciertas categorías sociales y las hicieron "fáciles de pensar". De igual modo, empezamos a sospechar de los vocabularios coloniales que se han liberado de manera subrepticia de sus vínculos históricos y reaparecen como nuestros conceptos explicativos de la práctica histórica y no como categorías populares que necesitan una explicación (Stoler, A. L., 2002).

La focalización de los estudios coloniales en tales tensiones del imperio, que fueron privadas y generales a la vez, ha colocado el sexo y los sentimientos no solo como metáforas del imperio, sino como sus elementos constitutivos (Stoler, A. L., I992a). La valoración de qué tanto lo personal es político ha renovado el ámbito de nuestros marcos archivísticos: los manuales para el hogar, la crianza de los niños y las guías médicas comparten el mismo espacio de documentos estatales clasificados, procesos legales e informes de comisiones como textos distintivos de las culturas coloniales de documentación. El enfoque en la cultura como un lugar de significado objetado, no compartido, propuesto por R. Williams, ha impulsado a los estudiantes de lo colonial a hacer lo mismo. Al dejar de contemplar la raza como cosa y verla como un conjunto poroso y proteico de relaciones, las historias coloniales se centran cada vez más en los puntos de empalme de las adscripciones archivadas y no archivadas para redefinir las subsunciones coloniales en un terreno más amplio (Chandler, J., Davidson, A. y Harootunian, H., 1994). Sin embargo, por más que la enmarquemos, la cuestión estriba en la lectura de los archivos con base en lo que tomamos como evidencia y lo que esperamos encontrar. ¿Cómo pueden los estudiosos del mundo colonial hacer una lectura "a contracorriente" de manera rápida y segura primero sin seguir la corriente? ¿Cómo podemos tomarlos a contracorriente sin adquirir antes un sentido de su textura y su granularidad? ¿Cómo podemos comparar los diferentes colonialismos sin conocer los circuitos de producción del conocimiento en los que funcionaban y las clasificaciones raciales en que reposan? Si una conceptualización de la etnografía colonial parte de la premisa de que la producción de archivos es en sí misma 
un proceso y una poderosa tecnología de gobierno, entonces se necesita más que ir en contra de las categorías archivísticas recibidas. Es necesario leerlos en busca de sus regularidades, de su lógica de remembranza, de sus densidades y distribuciones, de las consistencias de la desinformación, omisiones y errores, siempre siguiendo la corriente del archivo.

Se podría decir que asumir que conocemos tales aspectos reduce nuestras posibilidades de análisis. De esta manera, nos apoyaríamos con demasiada comodidad en historias predecibles con argumentos familiares. Apartaríamos nuestra atención de la influencia que las historiografías nacionalistas y los proyectos nacionales han tenido en la escritura de la histórica colonial. Esta posición olvida cuestionar la noción de que los Estados coloniales eran en principio y sobre todo máquinas ávidas de información en las que el poder se acrecentaba por la acumulación masiva de más información, mas no por su calidad.

Se daría por sentado que el arte colonial de gobernar estuvo motivado y alimentado por una ecuación reduccionista de la relación entre conocimiento y poder y que los Estados coloniales buscaban más conocimiento y más poder. Aún más, serían irrelevantes las propuestas fallidas, las visiones utópicas y los proyectos improbables porque "no fueron eventos". Una lectura de los archivos coloniales solo a contracorriente pasa por alto el poder en la producción de los archivos como tal.

\section{Civilidades y CREENCias EN LA PRODUCCIÓN DE ARCHIVOS}

i los documentos coloniales reflejan la supremacía de la razón,
también registran una economía emocional manifiesta en las
diferentes interpretaciones de lo que se imaginó, se temió, se
presenció y se escuchó de manera subrepticia. Esta lectura nos
lleva a las estructuras de sentimiento a las que los burócratas de
la colonia suscribían, al formulismo que cumplían, a la mezcla
de razón desapasionada, súplicas vehementes, textos culturales
y experiencias personales que establecían lo que decidían escri-
bir a sus superiores y luego se colocaba en los folios oficiales.
Los documentos coloniales holandeses registran esta economía
emocional de muchas maneras: en el afecto medido de los textos 
oficiales, en la crítica mordaz reservada para las notas al margen, en los pie de página de los informes oficiales donde se relegaban con frecuencia las evaluaciones de las prácticas culturales y se almacenaba el conocimiento local. Las preguntas ineludibles que formula S. Shapin en su historia social de la verdad podrían ser también las de los historiadores coloniales: ¿Qué se considera un fragmento de información creíble, a qué se le confirió virtud epistemológica y bajo qué criterio social? ¿Cuáles sentimientos y civilidades contribuyen al conocimiento colonial "experto" otorgado a personas con el derecho de proferir reivindicaciones de verdad fidedignas y no conferidas a otros?

Los archivos coloniales, como observa Echevarría, fueron repositorios legales de conocimiento y repositorios oficiales de políticas. Pero también fueron repositorios del buen gusto y la mala fe. Los escribas estaban encargados de realizar copias con finas plumas. Pero los informes sobre el orden colonial de las cosas dirigidos al Gobernador General en Batavia y al Ministro de las Colonias en La Haya solían elaborarlos hombres de letras, cuyo estatus en la jerarquía colonial se basaba tanto en la demostración de su educación europea como en su fingida ignorancia del conocimiento local, en sus habilidades para configurar los sucesos en argumentos familiares y su cultivo del arte de la deferencia, el disimulo y la persuasión. Todo se apoyaba en el uso sutil de su bagaje y bienes culturales. Como F. Colonna una vez señaló refiriéndose a la Algeria francesa, las políticas coloniales del conocimiento ponen en desventaja a aquellos con demasiado o insuficiente conocimiento de lo local (Colonna, F., 1997). En las Indias, la servidumbre civil con demasiado conocimiento de los asuntos javaneses era condenada por no apreciar las virtudes de la familiaridad limitada y selectiva.

En su minucioso estudio sobre el desarrollo de un sistema de inteligencia por el gobierno británico en la India, Ch. Bayly sostiene que el dominio del "conocimiento afectivo" fue una de las primeras preocupaciones del Estado colonial británico, que disminuyó durante el siglo XIX a medida que este se volvía más jerárquico y gobernar pasaba a ser un asunto rutinario (Bayly, C., I996). Sin embargo, se podría decir lo contrario: que el conocimiento afectivo estaba en el centro de la racionalidad política en esta forma colonial tardía. La modernidad colonial giraba en torno al disciplinamiento de sus representantes, sobre 
la vigilancia de la familia, en visiones orwellianas de intervención en la inculcación de la compasión, el desprecio y el desdén.

La acumulación de conocimiento afectivo no era, pues, un escenario ajeno a lo que con el tiempo iba a acaecer en los Estados coloniales. Los términos clave de los debates sobre blancos pobres y las prácticas de crianza de niños desde la década de 1930, justo antes del derrocamiento del gobierno holandés, lo hizo claro una y otra vez. Cuando los documentos coloniales clasificados se oponían al sostenimiento de los niños mestizos abandonados, es decir, que el "cuidado materno" (moederzorg) no se debía sustituir por el "cuidado estatal" (staatszorg), asignaban responsabilidad afectiva en el centro de sus proyectos políticos. Cuando estos mismos altos funcionarios escribían en uno y otro lado acerca de la mejor forma de despertar un "fuerte apego" por los Países Bajos entre la población europea local resentida, indiferente y creciente, "sentimiento" es la palabra que domina esta correspondencia. Puede que las autoridades holandesas nunca hayan estado de acuerdo en cómo cultivar la sensibilidad europea en sus jóvenes y solo imaginaron que ello era necesario en las primeras etapas del desarrollo infantil. Sin embargo, en el centro de estas deliberaciones sobre "la formación" y "la crianza" se encontraban reflexiones perturbadoras sobre lo que implicó hacer que alguien se "moviera" por un conjunto de regímenes sensoriales y distanciados de otros. Los Estados coloniales y sus autoridades, de manera similar a los metropolitanos, tenían una fuerte motivación por sus intereses permanentes en la distribución del afecto y un gran sentido de por qué esto era importante para la política colonial.

\section{LÓGICA CULTURAL Y CONVENCIONES ARCHIVÍSTICAS}

El archivo no tiene el peso de la tradición y no constituye la biblioteca de las bibliotecas, fuera de los límites de tiempo y lugar, sino que revela la norma de la práctica [...] el umbral de su existencia se establece mediante la discontinuidad que nos separa de lo que ya no podemos decir (Foucault, M., I972, p. 130). 
$\bigcup \int_{\mathrm{v}}^{\mathrm{n}}$ na manera de reconfigurar los usos que damos a los archivos coloniales es hacer una pausa y no pasar por alto sus convenciones, las prácticas que conforman su orden tácito, sus protocolos de organización y normas de disposición y referencia. Las convenciones pueden designar quiénes eran "fuentes” confiables, qué constituía evidencia "suficiente” y qué información (cuando no la hay) podía aportarse para elaborar un argumento creíble. Las convenciones proponen un consenso, pero no es claro que los expertos de los estudios coloniales las compartan en realidad. Las convenciones archivísticas se basaron en un conjunto cambiante de verdades coloniales acerca de lo que debe clasificarse como secreto y asunto de seguridad de Estado y I7. Sobre las distinciones administrativas entre "lo político" y "lo privado", y "lo criminal" frente a "lo subversivo", véase A. L. Stoler (1985 y I988). qué acciones se podrían descartar como aquellas que incitan a la venganza personal y a la cólera ad hoc o que se deban reconocer como subversión contra el Estado. ${ }^{17}$ Tales convenciones expusieron las taxonomías raciales y de gobierno, así como con cuánta habilidad, dificultad e irregularidad conocían las reglas del juego tanto burócratas experimentados como profesionales novatos.

La atención que se preste a estas convenciones puede llevar en dos direcciones: a la lógica consensual que inscriben y también, de manera más directa, a sus normas arbitrarias y los diversos puntos de desacuerdo. Los conflictos políticos se evidencian en la viabilidad cambiante de las categorías y los desacuerdos acerca de su uso. Pero como sugiere P. Starr, "la información fuera de lugar”, esto es, el fracaso de ciertas prácticas, percepciones y poblaciones para encajar en el sistema de clasificación preconcebido por el Estado, puede decirnos mucho más (Starr, P., I992). Se podría esperar que los comentarios acerca de las guarderías europeas en las colonias aparezcan en informes sobre educación, pero el hecho de que aparecieran continuamente en otras partes, como en informes sobre el reducción del pauperismo europeo y de los blancos pobres o en recomendaciones para acabar con el descontento del criollo, sugiere que aquello "fuera de lugar" con frecuencia era sensible y que eran los niños estimulados hacia las sensibilidades culturales equivocadas los que estaban peligrosamente fuera de lugar. 


\title{
LAS COMISIONES COLONIALES COMO HISTORIAS QUE LOS ESTADOS NARRAN PARA SÍ
}

\begin{abstract}
Como dice I. Hacking acerca de las categorías sociales, los archivos producían tanto como registraban las realidades que en apariencia solo describían. Narraron historias morales, establecieron precedentes en la búsqueda de evidencias y crearon historias cuidadosamente direccionadas. En ningún otro lugar se evidencia más esta labor de creación de historias que en la forma de la comisión de investigación o la comisión estatal. Por definición, las comisiones ordenaban el conocimiento, reorganizaban sus categorías y prescribían lo que debían saber los funcionarios estatales. Como advierte el antropólogo F. Husken acerca de las comisiones holandesas en la Java colonial: "Cuando nada más funcione y no se pueda llegar a una decisión, designar una comisión era una de las respuestas favoritas de las autoridades coloniales" (Husken, F., 1994, p. 2I3). Sin embargo, las comisiones no solo eran pausas en la política y tácticas de la dilación. Al igual que las estadísticas, ayudaban a "determinar [...] el carácter de los hechos sociales" y producían nuevas verdades a medida que producían nuevas realidades sociales (Hacking, I., I99I, p. I8I). Eran una respuesta a las crisis que generaba mayor ansiedad, al corroborar la realidad misma de tales crisis. ${ }^{18}$ En el tiempo en que la mayoría de las comisiones habían recorrido su camino (o dieron paso a la siguiente generación), podían ganar crédito al haber definido los "momentos cruciales", las justificaciones para las interven-

I8. Un buen ejemplo de lo que I. Hacking denomina "nominalismo dinámico" o el "efecto ciones y, no menos importante, el conocimiento experto.
\end{abstract} bucle" en la categorización.

I9. En otra parte hablaré acerca de las políticas de las comparaciones coloniales y, por lo tanto, no las trataré aquí. En una buena parte de mis escritos sobre los últimos I5 años de la construcción de las categorías raciales coloniales, me referí a la Comisión del Pauperismo en las Indias de 1902, a los comentarios sobre ella y a las investigaciones que la precedieron. La Comisión Carnegie de Sudáfrica y las investigaciones previas se comparan en un capítulo de mi próximo libro, Along the Archival Grain. En los textos (2000a y 2000b), se puede encontrar una discusión más general sobre las políticas de la comparación.
Las diversas comisiones instauradas para abordar el problema de los blancos pobres en las Indias entre las décadas de 1870 y principios de I900, y aquellas que se llevaron a cabo en Sudáfrica entre principios de la década 1900 y finales de 1920 son ejemplos de lo que tengo en mente. Existen ciertas características generales que comparten. ${ }^{19}$ Ambas produ- 
jeron volúmenes publicados y publicitados: Pauperism among the Europeans (19OI-I9O2) y The Problem of Poor Whites in South Africa (I929-I932). ${ }^{20}$ Las dos comisiones se designaron para tratar la indigencia de europeos y sus inapropiadas disposiciones
20. Los estudiosos del colonialismo podrían aparecer con muchos otros. Como ejemplo poco común de alguien que trata con la comisión como forma particular de conocimiento oficial, en este caso con la Comisión de Asuntos Nativos de Sudáfrica, véase A. Ashforth (I9g0). Véase también la discusión de F. Husken acerca de la Comisión de Bienestar de Java, cada vez más débil, citada en la nota al pie 62 .

frente al trabajo, la desigualdad racial, el dominio sexual y la moralidad. Cada una demandaba energía y pericia administrativa, implicaba varios años de trabajo, producía de miles de páginas y comprendía las observaciones de entrevistadores y de cientos de entrevistados. En el caso de las Indias, los cuestionarios de sondeo acerca de las uniones sexuales, los hijos ilegítimos y los acuerdos domésticos despertaban la ira de cientos de europeos coloniales, que condenaban el gobierno de las Indias como un "Estado inquisitorial". Dichas comisiones eran repositorios de las ansiedades coloniales: testimonios perturbadores sobre la inseguridad de los privilegios de los blancos, sobre las ambigüedades de la membresía en la categoría privilegiada de lo "europeo" y sobre la elaboración de una política de bienestar público firmemente basada en la raza. A ambas les preocupaba el número creciente de blancos empobrecidos porque les inquietaba algo más. Como se afirma en la Comisión Carnegie, "la proximidad de [...] las residencias" a los "no europeos" tendía a propiciar el contacto entre los nativos y los blancos, a "neutralizar la miscegenación", debilitando la línea del color y promoviendo la "igualdad social" (Comisión Carnegie, 1932, p. Xx.).

Estas comisiones se podrían y deberían leer por su extraordinario contenido etnográfico, pero también por el contenido que se evidencia en su forma. Al igual que otras comisiones coloniales, delimitan los grupos de personas que merecían los intereses y la inversión estatal. En segundo lugar, eran textos redentores, estructurados para ofrecer predicciones con base en relatos informales de exoneración y culpa. En tercer lugar, las dos comisiones fueron documentos para la creación de la historiografía y monumentos estatales por los cuales la historia era importante para los Estados imperiales y aquellos en proceso de consolidación. Al escribir el pasado, producían historias narrativas dramáticas con base en cronologías selectas, momentos decisivos y sucesos significativos. Al definir la pobreza del presente, también 
determinaban quiénes contarían como blancos en el futuro y, por ende, quiénes podían recibir ayuda del Estado.

Con todo lo anterior, escribieron, modificaron y reescribieron las genealogías raciales. Ninguna de estas comisiones fue la primera de su clase. Por el contrario, se hicieron creíbles por la manera como trazaban el pasado sobre las prescripciones del presente y las predicciones del futuro. También mostraban algo más. Cómo las prácticas sociales se circunscribían en eventos históricos y se convertían en cosas: cómo el aumento del desempleo y el empobrecimiento entre los colonos europeos se convirtió en un "problema" denominado "pobreza blanca" con características propias. Esta discriminación definía, a nivel fisiológico y psicológico, los diferentes tipos de personas, con maneras compuestas de "existir en el mundo" y disposiciones y estados mentales específicos. Al igual que otras comisiones coloniales, estas eran productoras consumadas de tipos y categorías sociales.

Las comisiones y las estadísticas eran elementos del arte de gobernar similares en varios aspectos. Ambas se inventaron en el siglo XVIII y las consolidó el

2I. La historia de las comisiones reales es más extensa aún. Véase, por ejemplo, D. Loades (1997). Sobre las estadísticas y la construcción del Estado, véase A. Desrosieres (I998. Sobre el siglo XX, véase W. J. Breen (I994). Estado liberal del siglo XIX. ${ }^{21}$ Las dos fueron productos y representaciones de la inversión estatal en la rendición de cuentas del sector público. Sin embargo, las comisiones exigían mayor autoridad moral ya que pretendían vigilar las prácticas estatales, revelar errores burocráticos y producir nuevas verdades acerca de los trabajos del Estado. Asimismo, estas comisiones de "blancos pobres" eran la quintaesencia de los productos de las "tecnologías biopolíticas". No solo establecieron la relación entre padres e hijos, niñeras y niños para la seguridad del Estado; también buscaron las pruebas etnográficas, los testimonios oculares de observadores-participantes de lo que hacían las personas (ya usaran zapatos, holgazanearan en los cobertizos, hablaran en holandés o malayo o hicieran que sus hijos oraran en la mañana). Estas eran prácticas directamente relacionadas con la revisión del Estado de su propia factibilidad.

Comisiones y estadísticas fueron parte de la "ciencia moral" del siglo XIX que cifraron y enumeraron las patologías de la sociedad. Mientras las estadísticas se valieron de las desviaciones de la media para identificar las desviaciones de la norma, las 
comisiones se sumaron a estas cifras a través de narraciones seleccionadas de "casos" individuales para medir las gradaciones de la moralidad..$^{22}$ Las comisiones a su vez afirmaron la autoridad estatal para establecer juicios sobre lo que se consideraba el bien moral y colectivo de la sociedad. Ambas eran herramientas prescriptivas y probabilísticas cuyo poder residía en parte en su capacidad de predecir y de desviar las posibilidades políticamente peligrosas.

$\mathrm{Al}$ igual que las estadísticas, la comisión demostró el derecho del Estado de ejercer su poder a través de su voluntad de verdad. En las Indias, la Comisión del Pauperismo le confirió autoridad moral al Estado al demostrar su conciencia moral y cohibición desinteresada, su disposición y compromiso para reflexionar de manera crítica sobre sus propios percances, para buscar la verdad "a cualquier costo". Sin embargo, su poder no solo residía en el cálculo del pulso moral del presente y sus implicaciones para el futuro. La comisión justificaba su licencia para invertir fondos, tiempo y fuerza de trabajo en parte mediante la repetición del pasado y recordando y acordando a sus lectores su valor perdurable. Las narraciones históricas dan forma a estos textos con relatos que desvían las causas de las privaciones y desigualdades que se alejan del presente, aún cuando repitan la carga permanente de las primeras políticas de las administraciones anteriores.

Por último, estas comisiones eran la quintaesencia de las tecnologías "cuasi estatales", parte del Estado y no, producto de los representantes del Estado, pero siempre conformadas por miembros externos. Si los Estados modernos cobraban parte de su fuerza al crear y mantener una barrera infranqueable entre ellos y la sociedad civil, como ha sostenido T. Mitchell, tales comisiones son ejemplo de este proceso. ${ }^{23}$ Sus asuntos específi-

22. Véase la discusión de A. Appardurai (I996) sobre la representación numérica en la India colonial como "clave de la normalización de la patología de la indiferencia".

23. Véase la discusión de A. Gramsci (I972) y T. Mitchell (I99I). cos eran generados por el Estado, pero con frecuencia los investigaban y escribían aquellos que no eran empleados permanentes. Tanto las comisiones de la Indias como la Carnegie delegaron organismos de expertos equipados para evaluar la moralidad (expertos religiosos), las desviaciones (abogados, educadores) y las enfermedades (médicos), a los que el Estado les otorgaba voz y autoridad pública de corto plazo sobre temas específicos. Ellos representaban las formas en que el Estado ejercía su voluntad de 
poder al convocar a autoridades expertas "externas" para verificar la capacidad del Estado para representar el interés público y su compromiso con el bien público.

\section{LA SEDUCCIÓN ARCHIVÍSTICA Y SECRETOS DE ESTADO}

omo los archivistas son los primeros en observar, para com-
prender un archivo es necesario comprender las instituciones
que ha servido. Qué temas tienen referencias cruzadas, qué
partes son reescritas y qué citas se traen a colación no solo nos
hablan acerca de las decisiones que se toman, sino cómo las his-
torias coloniales se escriben y reelaboran. La información fuera
de lugar subraya las categorías que importan, cuáles comienzan
a ser parte del sentido común y luego pierden aceptación. Asi-
mismo, ofrecen un conjunto de directrices hacia las ansiedades
que eluden formas más articuladas.

La comisión es una clase de convención archivística, los "secretos de Estado" son otra. Los Estados trafican con la producción de secretos y su diseminación selectiva. En este sentido, el Estado

24. G. Simmel escribió que "el desarrollo histórico de la sociedad es categorizado en muchos aspectos por el hecho de que aquello que en un comienzo era manifiesto ahora entra bajo la protección de lo secreto; y, por el contrario, aquello que una vez fue secreto, no necesita más este tipo de protección, sino que se revela" (Wolff, K., I95O, p. 33I). colonial holandés fue talentoso en esta actividad. ${ }^{24}$ Como afirmó M. Weber, el "secreto oficial" fue una invención específica de la burocracia que lo "defendía con fanatismo". Las designaciones "secreto", "muy secreto" y "confidencial" registraban más que ficciones del acceso denegado y el acceso público. Tampoco señalaban en mayor parte los problemas políticos delicados del Estado colonial. Al contrario, y más importante aún, estos códigos de ocultación eran aspectos del Estado mismo convertidos en fetiches. Los secretos de Estado designaban y generaban conocimiento y lectores privilegiados y, a la vez, recordaban a estos últimos qué conocimientos debía desearse y qué era importante saber. El informe secreto, como las comisiones, creaba categorías que no buscaban más que ofrecer una descripción. En las Indias, los documentos clasificados ejercían un peso político que exigía la participación de la policía secreta, informantes pagados y expertos. 
Los secretos implicaban acceso restringido, pero lo más notable en los archivos coloniales holandeses es que raras veces los elementos clasificados como "confidenciales" (vertrouwelijk, zeer vertrouwelijk, geheim, y zeer geheim) constituían secretos. Con seguridad, algunos documentos tenían que ver con la policía clandestina y las tácticas militares (como la preparación de los movimientos de las tropas para proteger de algún ataque a los colonos), pero muchos más trataban sobre elementos prosaicos y públicos de la vida en las Indias. ${ }^{25}$ Si se pudiera sostener que los documentos en que se registraba la presencia perturbadora de mendigos europeos e indigentes holandeses en las calles de Batavia eran "secretos" para los lectores residentes en la metrópoli, esta presencia no era un secreto para la mayoría de los europeos que vivían en los centros urbanos de las colonias.

La parte "clasificada" de estos informes no era su objeto (en este caso, los europeos indigentes de "sangre pura" y sus descendientes mestizos) sino el conflicto entre los funcionarios acerca de cómo actuar frente al problema, las diversas apreciaciones sobre la causa y cuántos indigentes había. Algunos informes eran "clasificados" porque los funcionarios no podían ponerse de acuerdo en si había 29 mestizos en situación de miseria o decenas de miles. ${ }^{26}$ En resumen, en ocasiones los documentos se clasificaban como "sensibles" y "secretos" por la magnitud del problema; otra veces porque los funcionarios no podían estar de acuerdo en dónde residía el problema. Sin embargo, quizá lo más sorprendente es el rango de la información confidencial que los estudiosos de lo colonial

25. Algemeen Rijksarchief (La Haya) Ministerie van Kolonien. Geheim No. II44/2284. Del Departamento de Justicia al Gobernador General, Batavia, 29 de abril de 1873 .

26. Algemeen Rijksarchief. Verbaal 28 de marzo de I874, no. 47. Del Departamento de Justicia al Gobernador General. esperan divulgar. Los secretos de Estado no son necesariamente verdades ocultas acerca del Estado, sino promesas compartidas de mantener ciertas confidencias. Si los secretos estatales son más que todo anotaciones cuidadosas que convenciones de ocultación, entonces cómo se producían los secretos de Estado o qué se consideraba secreto en un momento y no en otro puede indicar los términos variables de lo que se consideraba "sentido común”, así como los cambios en la racionalidad política. Como sostiene M. Ventresca en su estudio sobre por qué y cuándo cuentan los Estados, la información estadística en el siglo XVIII se consideraba fuente de poder estatal y, en consecuencia, no se 
publicaba. El acceso público a las estadísticas del Estado fue un fenómeno del siglo XIX (Ventresca, M., I995, p. 50). Los secretos de Estado conformaban un aspecto básico del archivo colonial, un elemento indicativo de la producción de ficciones del acceso desplegado por su contenido así como por su forma.

\section{LOS ARCHIVOS COLONIALES COMO "SISTEMA DE EXPECTATIVAS”}

ceptar la invitación de J. y J. Comaroff de "crear unos nuevos archivos coloniales por nosotros mismos" no solo implica atender los nuevos tipos de fuentes, como ellos señalan con acierto, sino diferentes maneras de abordar las existentes, diferentes maneras de leer lo que ha ya se ha hecho (Comaroff, J. y J., I992). El cambio de un proyecto extractivo a uno más etnográfico conlleva a que nuestras lecturas deban tomar nuevos rumbos a través de los archivos, tanto a lo largo de sus líneas de falla como a contracorriente. M. de Certeau definió la ciencia de la historia como una redistribución en el espacio, como el acto de transformar algo en otra cosa. Nos advierte que nuestros trabajos históricos en los archivos deben hacer más que "adoptar simplemente las clasificaciones anteriores"; deben alejarse de las limitaciones de la "serie $\mathrm{H}$ en los Archivos Nacionales" reemplazándolas con nuevos "códigos de reconocimiento" y "sistemas de expectativas" propios. Una estrategia como esta depende realmente de aquello que ya creemos saber (De Certeau, M., I988, p. I07). Para los estudiosos de lo colonial, tales códigos de reconocimiento y sistemas de expectativa están en el corazón de lo que aún se necesita aprender sobre las políticas coloniales. La amplitud de referencias globales y el alcance de la visión lateral que los regímenes coloniales alcanzan de manera irregular sugiere que una sensibilidad etnográfica, más que una actitud extractiva, puede ser más adecuada para identificar la manera como las naciones, imperios y regímenes racistas tomaron forma; no en las maneras en que se presenta el conocimiento seguro y el saber-hacer profesional, sino en formas perturbadoras y expectantes. 


\section{ReFERENCIAS}

Algemeen Rijksarchief (La Haya) Ministerie van Kolonien. Geheim No. II44/2284. Del Departamento de Justicia al Gobernador General, Batavia, 29 de abril de I873.

Algemeen Rijksarchief Verbaal 28 de marzo de i874, No. 47. Del Departamento de Justicia al Gobernador General.

Amin, S. (I995). Event, Metaphor, Memory: I922-I992. Berkeley: University of Michigan Press.

Anderson, B. (I99I). Census, Map, Museum. En Imagined Communities:Reflections on the Origin and Spread of Nationalism (pp. I63-I86). 2da ed. Nueva York: Verso.

Appardurai, A. (I996) Number in the Colonial Imagination. En Modernity at Large: Cultural Dimensions of Globalization (pp. II4-I38). Minneapolis: University of Minnesota.

Ashforth, A. (I990). The Politics of Official Discourse in Twentieth-Century South Africa. Oxford: Clarendon Press.

Bayly, C. (1996). Empire and Information: Intelligence Gathering and Social Communication in India, I780-I870. Cambridge: Cambridge University Press.

Berner, R. (1983). Archival Theory and Practice in the United States: An Historical Analysis. Seattle: University of Washington Press.

BreEn, W.J. (I994). Foundations, Statistics, and State-Building. Business History Review, 68, 45I-482.

Chandler, J., A. Davidson y H. Harootunian (eds.) (ig94). Questions of Evidence: Proof, Practice and Persuasion across the Disciplines. Chicago: University of Chicago Press.

Cohen, D. W. (I992). Burying SM: The Politics of Knowledge and the Sociology of Power in Africa. Portsmouth, NH: Heineman.

CoHn, B. (I980). History and Anthropology: The State of Play. Comparative Studies in Society and History, 22(2), I98-221.

Colonna, F. (1997). Educating Conformity in French Colonial Algeria. En Cooper, F. y Stoler, A. L. (eds.). Tensions of Empire: Colonial Cultures in a Bourgeois World (pp. 346-370). Berkeley: University of California Press.

Comaroff, J. y J. (I992). Ethnography and the Historical Imagination. Boulder: Westview Press.

Comisión Carnegie (I932). The Poor White Problem in South Africa, Report of the Carnegie Commission. Stellenbosch: Pro Ecclesia Drukkerij. 
Cook, T. (I997). What is Past is Prologue: A History of Archival Ideas Since I898, and the Future Paradigm Shift. Archivaria, 43.

Cook, T. (I994). Electronic Records, Paper Minds: The Revolution in Information Management and Archives in the Post-Custodial and Post-Modernist Era. Archives and Manuscripts, 22(2), 300-329.

CoOK, T. (I992). Mind over Matter: Towards a New Theory of Archival Appraisal. En Craig, B. (ed.). The Archival Imagination: Essays in Honour of Hugh A. Taylor (pp. 38-69). Ottawa: Asociación de Archivistas Canadienses.

Coombe, S. (I994). Archives Interdites: Les peurs françaises face a l'Histoire contemporaine. Paris: Albin Michel.

Cooper, F. y Stoler, A. L. (eds.) (I997). Tensions of Empire: Colonial Cultures in a Bourgeois World. Berkeley: University of Michigan Press.

Davis, N. Z. (I987). Fiction in the Archives: Pardon Tales and Their Tellers in Sixteenth-Century France. Stanford: Stanford University Press.

De Certeau, M. (I988).The Historiographic Operation. En The Writing of History. (pp. 56-II4). Nueva York: Columbia University Press..

Dening, G. (I995). The Death of William Gooch: A History's Anthropology. Honolulu: Hawaii University Press.

DerridA, J. (I995). Archive Fever: A Freudian Impression. Chicago: Chicago University Press.

Desrosieres, A. (I998) The Politics of Large Numbers: A History of Statistical Reasoning. Cambridge: Harvard University Press.

DiRks N. (ed.) (I992). Colonialism and Culture. Ann Arbor: University of Michigan Press.

Dirks N., Eley, G. y Ortner, S. B. (eds.). ([1983] I994). Culture, Power, History: A Reader in Contemporary Social Theory. Princeton: Princeton University Press.

DiRKS, N. (I993) Colonial Histories and Native Informants: Biography of an Archive. En Breckenridge, C. A.y Van der Veer, P. (eds.). Orientalism and the Postcolonial Predicament: Perspectives on South Asia (pp. 279-313). Philadelphia: University of Pennsylvania Press.

Duchein, M. (1992). The History of European Archives and the Development of the Archival Profession in Europe. American Archivist 55, I4-25.

Edwards, E. (I997) (ed.). Anthropology and Colonial Endeavour. The History of Photography 2I(I).

Evans-Pritchard, E. E. (I966). Social Anthropology: Past and Present, The Marett Lecture, I950. En Social Anthropology and Others Essays (pp. I39-I54). New York: Free Press. 
Evans-Pritchard, E.E. (I96I). Anthropology and History. Manchester: Manchester University Press.

Faubion J. (1993). History in Anthropology. Annual Review of Anthropo$\log , 22,35-54$.

Foote, K.E. (I990). To Remember and Forget: Archives, Memory, and Culture. American Archivist 53,(3), 378-393

Foucault, M. (I972). The Archaeology of Knowledge. New York: Pantheon Books.

Foucault, M. ([I97I] I977). Nietzsche, Genealogy, History. En Bouchard, D. (ed.). Language, Counter-Memory, Practice: Selected Essays and Interviews by Michel Foucault (pp. I39-I64). Ithaca: Cornell University Press.

Fox R. (I99I). For a Nearly New Culture History. En Fox, R. G. (ed.). Recapturing Anthropology: Working in the Present (pp. 93-II4). Santa Fe: School of American Research Press.

GEARY, P. (I994). Phantoms of Remembrance: Memory and Oblivion at the End of the First Millennium. Princeton: Princeton University Press.

Ginzburg, C. (I982). The Cheese and the Worms: The Cosmos of a Sixteenth-Century Miller. Londres: Penguin.

GinzBurg, C. (I989a). Clues: Roots of an Evidential Paradigm. En Ginzburg, C. Clues, Myths and the Historical Method (pp. 96-I25). Baltimore: Johns Hopkins University Press.

GinzBURG, C. (I989b). Clues, Myths, and the Historical Method. Baltimore: Johns Hopkins University.

GonzÁlez Echevarría, G. (I990). Myth and Archive: A Theory of Latin American Narrative. Cambridge: Cambridge University Press.

Grafton, A. (1997). The Footnote: A Curious History. Cambridge, MA: Harvard University Press.

Gramsci, A. (I972). Estado y la sociedad civil. En Hoare, Q.y Smith, G. (eds.). Selections from the Prison Notebooks of Antonio Gramsci (pp. 257-264). Londres: Lawrence and Wishart.

GuHA, R. ([I983] I994). The Proses of Counter-Insurgency. En Dirks, N., Eley, G. y Ortner, S. B. (eds.). Culture, Power, History: A Reader in Contemporary Social Theory (pp- 336-37I). Princeton: Princeton University Press.

Hacking, I. (I990). The Taming of Chance. New York: Cambridge University Press.

Hacking, I. (I99I). How Should We Do the History of Statistics? En Burchell, G., Gordon, C. y Miller, P. (eds.). The Foucault Effect: Studies in Governmentality (pp. I8I-I96). Chicago: Chicago University Press. 
Husken, F. (I994). "Declining Welfare in Java: Government and Private Inquiries, I9O3-I9I4”. En Cribb, R. (ed.). The Late Colonial State in Indonesia. (pp. 2I3.228). Leiden: KITLV.

LaCAPRA, D. (I995). History, Language, and Reading. American Historical Review, Ioo (3).

Levi-Strauss, C. (1966). The Savage Mind. Chicago: Chicago University Press.

LoAdes, D. (1997). The Royal Commissions. En Loades, D. Power in Tudor England (pp. 70-82). New York: St. Martin's Press.

McDonald, T. J. (ed.) (Ig66). The Historic Turn in the Human Sciences. Ann Arbor: University of Michigan Press.

Mitchell, T. (I99I). The Limits of the State. American Political Science Review, 85, 77-96.

Norris, C. (1995). Truth, Science, and the Growth of Knowledge. New Left Review, 210, I05-I23.

Nuttall, S. y Coetzee, C. (eds.) (1998). Negotiating the Past: The Making of Memory in South Africa. Cape Town, Oxford University Press.

O’Toole, J. M. (I994). On the Idea of Uniqueness. American Archivist, $57,(4), 632-659$.

Patriarca, S. (1998). Numbers and Nationhood: Writing Statistics in Nineteenth-Century Italy. Cambridge y Nueva York: Cambridge University Press.

Poovey, M. (1998). A History of the Modern Fact: Problems of Knowledge in the Sciences of Wealth and Society. Chicago: Chicago University Press

Posner, E. ([I984] I940). Some Aspects of Archival Development Since the French Revolution. En Daniels, M. y Walch, T. (eds.). A Modern Archives Reader (pp. 3-2I). Washington, D.C.: Servicio Nacional de Archivos y Registros.

Price, R. (1998). Convict and the Colonel: A Story of Colonialism and Resistance in the Caribbean. Boston: Beacon Press.

RAPPAPORT, J. (1994). Cumbe Reborn: An Andean Ethnography of History. Chicago: University of Chicago Press.

Richards, T. (1993). The Imperial Archive: Knowledge and the Fantasy of Empire. London: Verso.

Sekula, A. (I986). The Body and the Archive. Octubre 39, 3-64.

Shapin, S. (I994). A Social History of Truth: Civility and Science in Seventeenth-Century England. Chicago: Chicago University Press. 
Sherron De Hart, J. (I993). Oral Sources and Contemporary History: Dispelling Old Assumptions. The Journal of American History, 80,3, 582-595

Sider G. y G. Smith (eds.) (I997). Between History and Histories: The Making of Silences and Commemorations. Toronto: Toronto University Press.

Smith, B. G. (I995). Gender and the Practices of Scientific History: The Seminar and Archival Research in the Nineteenth-Century. American Historical Review IOO(4-5), II5O-II76.

StarR, P. (I992). Social Categories and Claims in the Liberal State. En Douglas, M.y Hull, D. (eds.). How Classification Works: Nelson Goodman among the Social Sciences (pp. 154-I79). Edinburgh: Edinburgh University Press.

Stoler, A. L. (I985). Perceptions of Protest: Defining the Dangerous in Colonial Sumatra. American Ethnologist, I2(4), 642-658.

Stoler, A. L. (I988). Labor in the Revolution. Journal of Asian Studies $47(2), 227-247$.

Stoler, A. L. (I992a). Sexual Affronts and Racial Frontiers. Comparative Studies in Society and History 34(3), 5I4-55I.

Stoler, A. L. (I992b). In Cold Blood: Hierarchies of Credibility and the Politics of Colonial Narratives. Representations 37, I5I-I89.

Stoler, A. L. (1995). Capitalism and Confrontation in Sumatra's Plantation, I870-I979. Ann Arbor: University of Michigan Press.

Stoler, A. L. (1996). Ethnography in the Archives: Movements on the Historic Turn. L.H. Morgan Lectures. University of Rochester.

Stoler, A. L. (I997). Racial Histories and Their Regimes of Truth. Political Power and Social Theory II, I83-255.

Stoler, A. L. (200I). Tense and Tender Ties: American History meets Postcolonial Studies. Conferencia pronunciada frente a la Organization of American Historians, Abril.

Stoler, A. L. (20oob). Beyond Comparison: Colonial Statecraft and the Racial Politics of Commensurability. Conferencia pronunciada frente a la Australian Historical Association, Adelaide, Julio.

Stoler, A. L. (2002). Genealogies of the Intimate. En Stoler, A. L. Carnal Knowledge and Imperial Power: Race and the Intimate in Colonial Rule. (pp. I-2I). Berkeley: University of Michigan Press.

Stoler A. L. y Strassler, K. (2000). Castings for the Colonial: Memory Work in 'New Order' Java. Comparative Studies in Society and History $42(\mathrm{I}), 4-48$. 
Troulllot, M. R. (1994). The Combing of History. Chicago: Chicago University Press.

Troulllot, M. R. (I995). Silencing the Past: Power and the Production of History. Boston: Beacon Press.

Ventresca, M. (I995). When States Count: Institutional and Political Dynamics in Modern Census Establishment, I800-I993. Unpublished PhD. Thesis. Stanford University.

White, H. (1987). The Content of the Form: Narrative Discourse and Historical Representation. Baltimore: Johns Hopkins.

White, L. (2000). Speaking with Vampires: Race and the Intimate in Colonial Rule. Berkeley: University of California Press.

WolfF, K. (ed.) (I950). The Sociology of George Simmel. Londres: Free Press.

Traducción al español de Josué Sierra Traductor profesional, Universidad del Rosario, Bogotá 